\title{
GABRIEL GALVEZ-BEHAR
}

\section{L'ÉTAT ET. L'INVENTION \\ UNE COMPARAISON FRANCO-ALLEMANDE DES CADRES DE LA PROPRIÉTÉ INDUSTRIELLE À LA FIN DU XIX ${ }^{\mathrm{e}}$ SIĖCLE}

Sommés de défendre les intérêts de leurs industries nationales, malmenés par la crise mais forts des théories économiques leur assignant une place nouvelle, les États du dernier quart du $\mathrm{XIX}^{\mathrm{e}}$ siècle contribuent à organiser leurs capitalismes nationaux en tenant compte des caractères particuliers de l'industrialisation qui s'opère alors. En effet, avec les évolutions nouvelles du système technique et l'affirmation croissante des liens entre la science et l'industrie, l'État doit porter une attention plus marquée à la promotion de l'invention et à la définition des droits de propriété industrielle'.

À cet égard, la comparaison franco-allemande apparaît comme un cas d'école. Forte de l'expérience initiée dans la chimie, l'industrie allemande tisse des liens nombreux avec une science conquérante et organisée par un État qui sait lui donner des impulsions décisives, tout en lui laissant une autonomie propice à la création. La France présente, de l'aveu même de ses savants, les travers d'un pays velléitaire, oubliant les initiatives précoces d'hommes comme Carnot, Gay-Lussac, voire Pasteur, qui avaient su, en leur temps, mettre la science au service de l'industrie.

Dans le dernier quart du $\mathrm{XIX}^{\mathrm{e}}$ siècle, cette opposition caricaturale mais récurrente semble s'appliquer à la propriété industrielle. À la France, consacrant depuis ses lois de 1791 et de 1844 le droit de l'inventeur, s'oppose l'Empire allemand, doté depuis 1877 d'une loi nouvelle sur les brevets et qui reconnaît la nécessité d'un examen préalable ou celle de licences obligatoires, autant d'éléments limitant les prérogatives de l'inventeur mais servant les intérêts plus généraux de l'industrie. Ce sont bien deux conceptions de l'État qui s'affrontent derrière cette opposition puisque la propriété industrielle définit les droits de l'inventeur au regard de l'intérêt général et puisque ces droits temporaires résultent d'une prérogative souveraine de l'État. Les débats autour de la définition des droits de la propriété industrielle contribuent donc à définir les contours de l'État, à lui assigner ou à lui dénier certaines prérogatives.

C'est sur cette définition concomitante des droits de l'inventeur et de l'État que nous souhaitons nous pencher, en comparant d'abord ses fondements juri-

\footnotetext{
1 Nous nous limiterons ici aux problèmes concernant les brevets d'invention, la propriété industrielle concernant plus largement, les brevets, les marques, les dessins et les modèles.
} 
diques, en identifiant ensuite les débats et ses acteurs et en décrivant, enfin, les pratiques en la matière.

Des fondements juridiques divergents

La définition et la garantie donnée par l'État aux brevets d'invention résultent de traditions juridiques qui, dans les cas français et allemand, sont fortement différenciées.

\section{Les hésitations d'un modèle libéral: la loi française de 1844}

Sans conteste, la législation française en matière de brevets d'invention est un héritage direct de la Révolution française qui établit de nouveaux rapports entre l'État et l'inventeur. Alors que les privilèges accordés aux inventeurs sous l'Ancien Régime dépendaient du pouvoir du souverain, les Constituants de 1791 consacrent le droit de l'inventeur en l'assimilant à un des droits de l'homme ${ }^{2}$. Dans le préambule de la loi du 7 janvier 1791, l'Assemblée nationale déclare considérer que stoute idée nouvelle dont la manifestation ou le développement peut devenir utile à la société, appartient primitivement à celui qui l'a conçue, et que ce serait attaquer les droits de l'homme dans leur essence que de ne pas regarder une découverte industrielle comme la propriété de son auteur ${ }^{3}$. En faisant dériver l'invention d'une idée qui ne pouvait qu'appartenir à son auteur, l'Assemblée nationale amenait à penser le droit de l'inventeur à la fois comme un droit naturel et comme une propriété inviolable et sacrée. La personnalité de l'inventeur se voyait donc protégée de l'arbitraire du souverain par ces deux principes complémentaires.

La loi de 1791 fut cependant l'objet de critiques pendant ses quelque cinquante ans d'application. Dès les années 1830 , un nouveau projet de loi vit le jour mais son adoption définitive dut attendre 1844. Les débats relatifs à l'adoption de la loi du 5 juillet 1844 furent cependant l'occasion de mettre à jour les faiblesses de la loi révolutionnaire. En effet, en prétendant garantir à l'inventeur »une pleine et entière jouissance« de sa propriété tout en limitant cette garantie dans la durée, cette dernière faisait reposer le droit de l'inventeur sur des fondements assez bancals. Philippe Dupin, député de l'Yonne,

2 Sur l'invention et les droits de l'inventeur sous l'Ancien Régime, cf. Liliane HILAIREPÉREZ, L'invention technique au siècle des Lumières, Paris 2000.

${ }^{3}$ Loi relative aux découvertes utiles, et aux moyens d'en assurer la propriété à ceux qui seront reconnus en être les auteurs, reproduite dans: Gérard EMPTOZ, Valérie MARCHAL, Aux sources de la propriété industrielle. Guide des archives de l'INPI, Paris 2003, p. 203. 
rapporteur du projet de loi sur les brevets d'invention en 1844, déclarait devant ses collègues:

L'un des caractères essentiels et dominants de la propriété, c'est la perpétuité. Celui qui est investi de ce droit ne peut le perdre que par une abdication ou par une expropriation avec indemnité préalable; ou bien ce n'est plus la propriété. L'Assemblée constituante avait ellemême proclamé ce principe dans l'article 17 de la déclaration des droits de l'homme.

Aussi fut-il nécessaire de donner à la nouvelle loi des bases inébranlables en assimilant le droit de l'inventeur non plus à une propriété mais à »un contrat sous la foi duquel le génie de l'invention livre à la société ses précieuses découvertes $\varkappa^{5}$. La loi sur les brevets d'invention de 1844 remplaça donc un droit de propriété par un droit exclusif d'exploitation.

Un tel changement ne signifiait toutefois pas que le droit de l'invention fût concédé par l'Etat, ni que l'inventeur fût placé sous la tutelle de ce dernier. La loi de 1844 affirme en effet dans son article $1^{\text {er. }}$ :

Toute nouvelle découverte ou invention dans tous les genres d'industrie confère à son auteur, sous les conditions et pour le temps ci-après déterminés, le droit exclusif d'exploiter à son profit ladite découverte ou invention.

Ce droit est constaté par les titres délivrés par le Gouvernement sous le nom de brevets d'invention. ${ }^{6}$

Bien qu'il ne soit plus un droit de propriété mais la contrepartie d'un contrat passé avec la société, le droit de l'inventeur n'apparaît pas comme un droit concédé par le gouvernement. La découverte et l'invention restent au fondement du droit de l'inventeur et par elles seules ce droit est conféré. Ainsi l'État n'intervient-il pas pour concéder un privilège mais pour permettre à l'inventeur de faire constater un droit. Par là même, l'esprit de la loi de 1791 qui consacrait un droit naturel reste préservé.

Ce point est important. Dans la perspective de la loi de 1791, le droit naturel de l'inventeur devait être automatiquement reconnu. Pour cette raison, et contrairement aux pratiques d'Ancien Régime, le brevet d'invention était délivré »sur simple requête au Roi, sans examen préalable «?. Parce qu'il bénéficiait d'un a priori positif, parce qu'il était présumé inventeur jusqu'à preuve du contraire, le requérant bénéficiait du brevet et, ce faisant, de la protection de l'État. La loi de 1844 maintient donc ce principe car, aux yeux de ses parti-

\footnotetext{
${ }^{4}$ Rapport fait au nom de la commission chargée de l'examen du projet de loi sur les brevets d'invention, par M. Philippe Dupin, député de l'Yonne, dans: Adrien HuARD, Répertoire de législation et de jurisprudence en matière de brevets d'invention, Paris 1863, p. 231 et suiv.

${ }^{5}$ Ibid. p. 237.

"Loi du 5 juillet 1844 sur les brevets d'invention, dans: EMPTOZ, MARCHAL, Aux sources, p. 206.

${ }^{7}$ Loi portant règlement sur la propriété des auteurs d'inventions et découvertes en tout genre d'industrie, dans: Ibid. p. 205.
} 
sans, il était impossible de trouver une autorité susceptible de décider parfaitement de la réalité d'une invention ou d'un perfectionnement. Aux tribunaux seuls, aux termes de débats contradictoires, devait revenir cette tâche difficile. Sur ces bases est assise l'intervention de l'État en matière de propriété industrielle. Afin de garantir le droit naturel de l'inventeur contre toute forme d'arbitraire, seul le pouvoir judiciaire peut intervenir pour juger de la validité des droits de chacun. L'Administration, quant à elle, doit se borner à enregistrer des déclarations et à les publier pour informer la société toute entière des progrès de la technique.

Toutes les ambiguités de la loi de 1791 ne sont pas effacées par la loi de 1844. La négation du droit de propriété fut l'objet de fortes critiques de la part de ceux qui virent là une atteinte au prestige de l'inventeur et un encouragement à la contrefaçon ${ }^{8}$. À la fin du XIX ${ }^{\mathrm{e}}$ siècle, la tradition faisant du droit de l'inventeur à la fois un droit naturel et un droit de propriété reste encore très forte. Lors du congrès international de la propriété industrielle tenu en 1878, la question de la nature de ce droit, de nouveau posée, fut résolue en ces termes: "le droit des inventeurs et des auteurs industriels sur leurs œuvres, ou des fabricants et négociants sur leurs marques est un droit de propriété; la loi civile ne le crée pas: elle ne fait que le réglementer «". Refusant de dire explicitement que le droit des inventeurs était un droit naturel, le congrès le laissait entendre de manière implicite et consacrait à nouveau le droit des inventeurs comme un droit de propriété.

Ce débat récurrent sur la nature du droit des inventeurs n'est pas vain au regard de la question qui nous préoccupe, car il possède une dimension indéniablement politique. Il détermine les rapports devant exister entre l'inventeur, la collectivité et l'État. Reconnaître à l'inventeur un droit naturel de propriété permet de lui donner accès au droit commun et lui confère, par conséquent, la protection dont bénéficie chaque citoyen du fait de la déclaration de 1789. Ce rapport à la Révolution française contribue d'ailleurs à renforcer la dimension politique de la question. Faire apparaître les droits de l'inventeur comme un héritage de 1791 permet de les présenter comme un acquis, voire un outil de la démocratie. Ainsi, pour lutter contre sune aristocratie toute puissante de la finance et de l'industrie, aristocratie bien autrement gênante et nuisible que l'aristocratie du nom et de la naissance «, le brevet était-il, aux yeux d'Eugène Pouillet, wune barrière contre laquelle les imprudentes convoitises de l'accapareur [viendraient] se briser ${ }^{10}$. Dans le cas français, les débats sur la propriété industrielle ne dérivent donc pas de considérations essentiellement économiques mais plutôt de questions politiques. La définition de la propriété industrielle découle en premier lieu de la construction d'un double rapport entre l'individu et la société, entre l'État et l'inventeur.

\footnotetext{
${ }^{8}$ HUARD, Répertoire, p. 262.

${ }^{9}$ Journal officiel de la République française, 24 septembre 1878 , p. 9393.

${ }^{10}$ Eugène POUILLET, Traité théorique et pratique des brevets d'invention et de la contrefaçon, Paris ${ }^{5} 1909$, p. XXIII. Eugène Pouillet est l'un des principaux juristes français en matière de propriété industrielle à la fin du $\mathrm{XLX}^{e}$ siècle.
} 


\section{Le modèle allemand, un modèle sans tradition}

À la différence de la France, l'Empire allemand de la fin du XIX ${ }^{\mathfrak{e}}$ siècle ne peut se prévaloir d'une tradition séculaire en matière de brevets d'invention. Avant l'adoption de la loi de 1877, coexistent en l'Allemagne 29 lois différentes reposant chacune sur des principes particuliers. Alors qu'en Prusse le publicandum de 1815 contraignait le requérant d'un brevet à un examen préalable sévère, le Palatinat bavarois appliquait la loi française de 1791, tandis que l'Alsace-Lorraine, conquise au cours de la guerre franco-prussienne, obéissait à celle de 1844. Dans l'ancien Land de Mecklembourg ou dans les anciennes villes hanséatiques de Brême et de Hambourg, toute invention divulguée tombait dans le domaine public".

Des progrès partiels furent accomplis avec l'avènement du Zollverein mais le caractère éclaté des législations rendait la protection des inventions particulièrement hasardeuse dans l'espace allemand. Les vices de ces législations engendrèrent un vif mouvement de contestation de la part des économistes libéraux à tel point qu'en 1868, Bismarck lui-même s'exprimait pour la suppression des brevets devant le conseil fédéral de la Confédération de l'Allemagne du Nord ${ }^{\prime 2}$.

L'avènement de l'Empire allemand et les prémisses de la grande dépression conduisirent à renverser l'opinion publique à l'égard des brevets. Afin de réaliser l'unité de l'Empire en matière de propriété industrielle et de protéger une industrie naissante, le Reichstag saisit dès 1872 le gouvernement impérial de la question. L'exposition de Vienne de 1873, et la tenue d'un premier congrès international de la propriété industrielle, la fondation d'une Association pour la défense des brevets à l'instigation de Werner Siemens, un an plus tard, furent autant de facteurs qui achevèrent de conforter les partisans d'une législation nouvelle. Dès lors, la première loi impériale sur les brevets d'invention, votée le 25 mai 1877 , entra en vigueur le $1^{\text {er }}$ juillet de la même année.

Ce tableau rapide souligne que »le droit des inventeurs a en Allemagne des origines récentes et une très courte histoire ${ }^{13}$. En 1877, l'Allemagne établit

" Charles LYON-CAEN, Loi sur les brevets d'invention du 25 mai 1877, dans: Annuaire de législation étrangère 8 (1878) p. 106.

${ }^{12}$ Cf. Alfred HeGGEN, Erfindungsschutz und Industrialisierung in Preussen 1793-1877, Göttingen 1975; Peter KURZ, Das Kaiserliche Patentamt im Patentgesetz 1877, dans: Mitteilungen der deutschen Patentanwälte 11/12 (2002) p. 487; Matthias-Hermann GEHM, Das württembergische Patentrecht in 19. Jahrhundert, thèse de doctorat, université de Sarrebruck 2001; David GILGEN, Entstehung und Wirkung des deutschen Patentsystems im Kaiserreich. Eine neoinstitutionalistische Analyse, thèse de doctorat, Florence 2003, à paraître; Margrit SECKELMANN, Industrialisietung, Internationalisierung und Patentrecht im Deutschen Reich 1871-1914, thèse de doctorat, Francfort/M. 2004, à paraitre; ID., Industrial Engineering and the Struggle for the Protection of Patents in Germany, 1856-1877, dans: Quaderns d'Historia de l'Enginyeria 5 (2002-2003) p. 299-306.

13 Joseph BONNET, Étude sur la législation allemande sur les brevets d'invention, Paris 1902,p. 1. 
les fondements de sa propriété industrielle dans un contexte radicalement différent des bouleversements de la Révolution française. En 1877, il ne s'agit pas pour le législateur allemand de proclamer un droit naturel de l'inventeur ou de lui conférer un statut inviolable et sacré, mais de construire un outil juridique efficace et favorable au développement de son industrie. Aussi n'est-il pas étonnant de voir combien les bases de la loi allemande de 1877 furent différentes de celles de la loi française alors en vigueur.

Les fondements théoriques de la loi allemande de 1877 contrastent, en effet, avec leurs pendants français. Pour résoudre l'aporie posée par la question de la propriété des inventions, certains juristes mirent en avant, dès les années 1870 , l'idée d'un droit des biens immatériels ${ }^{14}$ distinct du droit personnel, du droit réel ou de celui des créances ${ }^{15}$. De ce point de vue, le droit de l'inventeur ne découle pas de sa personne, ni d'un contrat passé entre l'inventeur et la société, mais du caractère immatériel de l'invention. En effet, l'invention procède alors d'une idée immatérielle qui »dès qu'elle [...] est passée dans nos conceptions et nos habitudes, devient, comme l'air et la lumière, res communis omnium éloignant par là-même la domination de l'individu «' ${ }^{16}$. Mieux, si la genèse de l'invention a un côté indéniablement individuel, elle a aussi un trait profondément collectif ${ }^{17}$ et le droit des brevets doit tenir compte autant des revendications de l'inventeur que de celles de la société.

Les conceptions allemandes en la matière trahissent donc la volonté de construire un système juridique susceptible de protéger non pas tant l'inventeur que la collectivité en général, en consacrant les droits de l'industrie avant tout. Cet impératif est d'autant plus fort que, loin d'être éternelle, la justification du droit de l'inventeur entend répondre aux contraintes sociales et historiques. Aussi les attentes de la grande industrie et du capital, qui permettent à la société de diffuser les bienfaits de l'invention, doivent-elles être satisfaites. Contrairement à la tradition française qui tend à asseoir les droits de l'inventeur sur des principes immuables, le modèle allemand se présente comme un modèle essentiellement pragmatique et dynamique.

Cette approche théorique sur le droit de l'inventeur met en lumière les divergences profondes qui existent alors entre le modèle français et le modèle allemand et explique, pour une part, le rôle différent dévolu à l'État. Cette dif-

\footnotetext{
${ }^{14}$ L'idée est défendue par Josef KOHLER pour la première fois en 1875 dans: Annalen der badischen Gerichte 41,1875 , p. 100 et suiv. En Belgique, le juriste E. Picard défend au même moment des iđées similaires.

${ }^{15}$ Gustave HUARD, De l'évolution du droit en matière de propriété intellectuelle dans: Annales de droit commercial 14 (1900) p. 206-207.

${ }^{16}$ Josef KOHLER, Forschungen aus dem Patentrecht, Mannheim 1888, p. 117.

${ }^{17}$ ID., Handbuch des deutschen Patentrechts in rechtsvergleichender Darstellung, Mannheim 1900, p. 6: "Die Erfindung ist nach ihrer Anlage wesentlich individualistisch; allein sie hat einen tief genossenschaftlichen Zug. Und so auch das Patentrecht.« Une étude plus détaillée serait cependant nécessaire pour tenir compte de l'évolution de la pensée de Kohler dans le dernier quart du $\mathrm{XDX}^{\mathfrak{e}}$ siècle.
} 
férence se lit particulièrement bien dans l'importance donnée à l'examen préalable en Allemagne.

La loi de 1877 et celle de 1891 entérinent en effet le principe d'un examen préalable et d'un appel aux oppositions à l'encontre d'une demande de brevets $^{18}$. L'examen préalable du brevet conduit les membres du Patentamt à s'assurer de la brevetabilité de l'invention tandis que l'appel aux oppositions permet à ceux qui contestent la prise de brevet de faire connaître leurs propres droits. En France, un tel principe avait été rejeté parce que l'on craignait que son application fût impraticable ou qu'elle donnât lieu à une censure industrielle. Par ailleurs, on déniait à l'autorité administrative le pouvoir de limiter un droit tenu comme naturel. Seuls les tribunaux devaient avoir une telle responsabilité. Dans le cas allemand, l'État en général et l'autorité administrative en particulier se voient imposer le devoir de procéder à un tel examen. Pour Kohler, c'est un devoir pour l'État de garantir la clarté et la sécurité à la fois aux inventeurs et aux industriels en traçant les limites de la propriété immatérielle $^{19}$. Tout comme il trace le cadastre, l'État doit songer à borner cette propriété originale.

Comparée à la législation française, la législation allemande en matière de brevet d'invention suppose donc une implication plus forte et plus franche de l'État. Cette différence ne tient cependant pas à des différences de mentalité. Comme on l'a vu, la législation sur les brevets en Allemagne a été fortement contestée avant les années 1870 . Ce sont les conditions de la genèse du droit allemand en la matière qui ont été déterminantes. Alors que le droit français dérivait d'une conception jus naturaliste née avec la Révolution française, le droit allemand résulte d'une double volonté de construire un droit en phase avec son temps ${ }^{20}$ et de le mettre au service d'une industrie en plein essor. L'État apparaît comme l'instrument nécessaire pour répondre aux défis du moment en donnant à l'industrie les moyens de promouvoir l'invention.

\section{L'espace des débats}

Le regard porté sur les fondements juridiques des modèles français et allemand en matière de propriété industrielle ne doit pas laisser penser que ces derniers demeurent fixés dans le marbre. Ils furent au contraire, tant en France

\footnotetext{
${ }^{18}$ Sur la loi de 1891 , cf. Joseph BONNET, Étude sur la législation et La nouvelle loi allemande sur les brevets d'invention, dans: La Propriété industrielle, $1^{\text {er }}$ mai 1891 , p. 58 et suiv.

${ }^{19}$ KOHLER, Handbuch des deutschen Patentrechts, p. 33: »Beim Patentwesen ohne Vorprüfung dankt der Staat ab; er verzichtet auf seine Aufgabe, Klarheit und Sicherheit zu schaffen, er überläßt das Industrieleben der Nacht und dem dichten Nebel.«

${ }^{20}$ Kohler ne déclare-t-il pas: $» \mathrm{Da} B$ ein solches Erfinderrecht nicht ewig sein kann, daß es der Zeit seinen Tribut zahlen, daß es nach bestimmter Zeit untergehen muß, versteht sich von selbst«, ibid. p. 7.
} 
qu'en Allemagne, l'objet de débats récurrents qui conduisirent à une redéfinition continue du rôle de l'État dans l'économie. De ces demiers résulta en Allemagne une modification de la loi de 1877; en France, les projets de réforme se succédèrent mais donnèrent lieu à des mesures jugées toujours incomplètes.

\section{L'État et la construction du débat public en Allemagne}

Dès les premières années de son application, la loi de 1877 fit l'objet d'un certain nombre de critiques: l'examen préalable fut jugé trop sévère tandis que naissait le désir de voir transformé le Patentamt en véritable instance juridique $^{21}$. Pour répondre à ces contestations, le Bundesrath décida le 8 juillet 1886 de provoquer une enquête sur la loi de 1877 et de former une commission à cet effet. Constituée de 33 membres, parmi lesquels l'on comptait aussi bien des représentants de la grande industrie ${ }^{22}$ que des juristes ou des hauts fonctionnaires $^{23}$, la commission d'enquête se prononça à la quasi-unanimité sur le maintien de l'examen préalable et porta toute son attention sur le problème des produits chimiques. Le rapport de la commission fit alors l'objet d'un projet de loi gouvernemental discuté par les chambres de commerce et les associations commerciales et industrielles dans le courant de l'année $1890^{24}$. Plusieurs d'entre elles se prononcèrent en faveur de la suppression de l'examen préalable et demandèrent que les taxes fussent abaissées.

Les partisans des réformes se réunirent alors à Berlin le $1^{\text {er }}$ décembre 1890 lors d'une grande conférence pour la protection de la propriété industrielle. Cette dernière ne se prononça qu'à la majorité d'une seule voix pour l'examen préalable et demanda, à son tour, l'institution d'un tribunal spécial en matière de brevets. Ses résolutions, transmises au Reichstag, ne furent que partiellement retenues. La loi du 7 avril 1891 réorganisa l'examen préalable en permettant à l'inventeur d'être entendu personnellement par les examinateurs lorsque la brevetabilité de son invention était contestée et introduisit des adoucissements concernant le paiement des taxes ${ }^{25}$.

L'adoption de la loi de 1891 ne parvint pas à faire taire les critiques. En 1899 , le député Möller, représentant la ville de Duisburg, affirmait que le Patentamt n'était pas en mesure de procéder à un examen fiable, faute de personnel. Quant au porte-parole de la Société des ingénieurs allemands, Riedler, il

${ }^{21}$ La Propriété industrielle, $1^{\text {er }}$ août 1886, p. 65 et suiv.

${ }^{22}$ On y trouve entre autres: W. Siemens, au titre de conseiller intime du gouvernement et de membre de l'Académie des sciences; Caro, directeur de la BASF; Klüpfel, représentant des usines Krupp.

${ }^{33}$ Cf. Stenographische Berichte über die Verhandlungen der Enquete in Betreff der Revision des Patentgesetzes vom 25 mai 1877, Berlin 1887.

${ }_{24}$ La Propriété industrielle, $1^{\text {er }}$ novembre 1890 , p. 130.

${ }^{25}$ La Propriété industrielle, $1^{\text {er }}$ mai 1891 , p. 58 et suiv. 
s'interrogea sur l'opportunité de maintenir un système aussi imparfait ${ }^{26}$. Au cours du congrès de la propriété industrielle tenu à Francfort en 1900, les membres de l'Association allemande pour la protection de la propriété industrielle déclarèrent que la loi sur les brevets du 7 avril 1891 contenait des défectuosités telles que sa révision paraissait nécessaire. En 1913, ces contestations conduisirent le gouvernement à proposer un projet de loi que la guerre empêcha d'aboutir. Ainsi, contrairement à ce que pourrait laisser penser une comparaison trop rapide, le modèle allemand n'eut donc rien de statique et les attributions de l'Etat en matière de propriété industrielle furent régulièrement discutées et contestées.

L'influence des intérêts industriels fut l'un des facteurs qui contribua à faire émerger la législation allemande sur les brevets. Ainsi la pression des groupes d'intérêts industriels dans les débats concernant la propriété industrielle se fit sentir dès les années 1870 , avec la fondation, en 1874, du Patentschutzverein par Werner Siemens, et plus particulièrement à propos des brevets dans l'industrie chimique.

L'importance de l'industrie chimique dans l'économie allemande conduisit, en outre, ses représentants à peser de tout leur poids dans la définition des nouvelles règles de la propriété industrielle. L'exemple de la France et, plus particulièrement, les conséquences des procès autour de la fuchsine qui avaient conduit un certain nombre de chimistes français à immigrer en Suisse, furent longuement médités par les industriels de la chimie allemande ${ }^{27}$. Bon nombre d'entre eux s'exprimèrent d'ailleurs contre la législation des brevets en la matière, craignant que l'Allemagne ne connaisse le même sort que la France.

À l'initiative des grandes firmes de l'industrie chimique, et notamment de Hœchst, un compromis avait été trouvé dans la loi de 1877 puisque les matières obtenues par des procédés chimiques furent déclarées non-brevetables ${ }^{28}$. Même si le procédé, lui, pouvait faire l'objet d'un brevet, cette disposition ne suffisait pas à répondre aux attentes de l'industrie chimique; elle fut au contraire la source de difficultés nouvelles. Elle favorisait, en effet, l'importation de produits chimiques fabriqués selon des procédés brevetés en Allemagne. La loi ne permettant pas d'assurer un monopole d'exploitation pour un produit chimique, de tels produits pouvaient concurrencer les produits allemands sans difficulté.

${ }^{26}$ La Proprièté industrielle, 30 avril 1899, p. 60.

${ }^{27}$ Dans les années 1860 , les détenteurs du brevet de la fuchsine, matière colorante synthétique, intentèrent des procès en contrefaçon à des concurrents ayant produit la même matière selon des procédés différents. La justice leur donna raison confirmant leur monopole sur un produit quel que soit le procédé de fabrication. Pour une comparaison entre la législation sur les brevets en matière de produits chimiques en France et en Allemagne, cf. Maurice GUÉRIN, Les aspects économiques de la législation des brevets d'invention, dans: L'industrie des produits chimiques, thèse pour le doctorat de droit, Paris 1922.

${ }_{28}^{28}$ Loi sur les brevets d'invention du 25 mai 1877, dans: Annuaire de législation étrangère 8 (1878) p. 110. 
L'industrie chimique s'émut de cette situation qui fit l'objet de nombreuses discussions au sein de la commission d'enquête de 1886. Caro, dirigeant de la BASF et président de la commission des brevets de la Société allemande pour la défense des intérêts de l'industrie chimique, intervint dans ses travaux pour obtenir des dispositions nouvelles favorables à son industrie. Ses propositions furent entendues puisque la loi de 1891 étendit l'effet du brevet d'invention aux produits obtenus directement par un procédé unique.

L'État se montra également à l'écoute des revendications des différents acteurs de l'industrie allemande dans un autre domaine: celui des inventions d'employés. Avec les progrès de la recherche industrielle dans les grandes entreprises allemandes, il devenait en effet toujours plus difficile de savoir qui du patron ou du salarié détenait les droits sur l'invention. Alors que les représentants de la grande industrie avaient toujours réussi à imposer le principe du premier déclarant ${ }^{29}$, la montée en puissance et l'organisation de la profession d'ingénieur vint troubler cet équilibre favorable à l'industrie. À partir de 1904, avec la formation d'une association, le Bund der technisch-industriellen Beamten, les revendications des ingénieurs se firent de plus en plus fortes. Dès 1906, le Reichstag fut saisi d'une demande de modification de la loi qui aboutit, en juillet 1913, à un projet gouvernemental affirmant la volonté d'abandonner le principe du premier déclarant et celle d'établir une juste compensation pour l'inventeur-employé. Cette réforme fut cependant interrompue par la guerre $^{30}$.

Les débats sur la propriété industrielle en Allemagne firent donc intervenir les acteurs principaux de l'industrie. Il ne faudrait pas pour autant concevoir l'État allemand comme la caisse d'enregistrement des grands intérêts: les réactions du Reichstag aux résolutions du congrès de 1890 soulignent, si besoin en était, le caractère négociable des prises de décision en la matière. En outre, l'accueil fait aux protestations des ingénieurs au début $d u X X^{\mathfrak{e}}$ siècle empêche de considérer l'État comme le porte-parole des seuls grands intérêts.

L'originalité du rôle tenu par l'État en Allemagne concernant cette question tient sans doute à sa capacité d'organiser les débats et de donner aux représentants de l'industrie une légitimité à parler en son nom sur ce point précis. À cet égard, la commission de 1886 est assez exemplaire: sous la pression d'un certain nombre de groupes, le gouvernement réunit une commission ad hoc, où se trouvent représentés les acteurs de l'industrie allemande dont le travail constitue la base du processus législatif. Pour que le droit fût réellement au service de l'industrie, n'était-il pas préférable que cette dernière eût effectivement voix au chapitre?

\footnotetext{
${ }^{29}$ Selon ce principe, le brevet est accordé à la première personne déposant la demande de brevet, qu'elle soit l'inventeur réel ou non. À l'époque, ce même principe prévaut en France contrairement aux États-Unis qui appliquent le principe du premier inventeur.

${ }^{30}$ Sur ce point, cf. Kees GISPEN, New Profession, Old Order, Engineers and German Society, 1815-1914, Cambridge 1989, p. 255 et suiv.
} 


\section{La difficile représentation des intérêts de l'inventeur en France}

En France aussi, les débats concernant la propriété industrielle sont récurrents à la fin du $\mathrm{XIX}^{\mathrm{e}}$ siècle. Dès 1883 , la prolongation de la durée du brevet était réclamée et en 1888, le Syndicat des inventeurs de France demandait qu'une réforme totale de la législation de 1844 fût initiée. Pour les auteurs du projet, les différentes propriétés intellectuelles n'avaient pas à être dissociées et, de ce fait, un droit de propriété aussi étendu que celui de la propriété littéraire et artistique devait être accordé à l'inventeur. Le gouvernement se soucia de prendre en compte les avis des acteurs économiques en procédant à une enquête auprès des chambres de commerce, notamment. La Chambre de commerce de Paris refusa de voir remis en question les fondements d'une loi qui avait pour elle »la sanction de l'expérience ${ }^{31}$. Elle accepta, en revanche, des retouches comme l'instauration d'une taxe progressive et non plus fixe, la simplification des procédures de demande ainsi que la publication intégrale et par fascicule séparé des brevets délivrés.

Le ministère du Commerce et de l'Industrie interrogea aussi les professionnels de la propriété industrielle, à commencer par les agents de brevet. Ces derniers examinèrent les problèmes posés par une modification de la loi de 1844 en général, et par la question de l'examen préalable en particulier. Sur cette question, le Syndicat des ingénieurs et conseils en matière de propriété industrielle se montra lui-même fort divisé. Alors que Charles Thirion, organisateur du congrès de 1878 sur la propriété industrielle, se prononçait pour l'examen préalable, Dominique-Antoine Casalonga déclarait:

La faculté créatrice de l'inventeur est la plus haute expression de l'initiative individuelle. Pourquoi mettre l'inventeur en tutelle et vouloir l'assister d'une Administration-Providence, faillible sous tant de rapports, et qui ne peut même offrir une sanction à la responsabilité qu'elle encourt en cas d'erreur? ${ }^{32}$

La consultation des différents intéressés ne put donc aboutir à des conclusions claires. En 1892, le Syndicat des ingénieurs-conseils réitéra ses demandes en vue d'un changement de la loi de 1844 et fit parvenir différentes pétitions auprès de la Chambre des députés et du Sénat. Là encore, bien que le gouvernement convînt d'une nécessité de modifier la loi, aucune disposition ne fut prise.

Comment expliquer alors cette inertie du gouvernement face à une demande réitérée et perçue comme légitime? Comme en Allemagne, le gouvernement procéda à une enquête auprès de ceux qui étaient susceptibles d'être intéressés

\footnotetext{
${ }^{31}$ Archives de la Chambre de commerce et d'industrie de Paris [ACCIP], délibérations de la Chambre de commerce de Paris, séance du 20 juin 1888.

${ }^{32}$ Bulletin de la Société des ingénieurs et conseils en matière de propriété industrielle, séance du 26 novembre 1888 .
} 
par les réformes. Cependant, contrairement au cas allemand, ces dernières émanèrent avant tout de professionnels, qu'il s'agisse d'ingénieurs-conseils ou d'avocats, et à bien des égards la question de la propriété industrielle en France apparut comme une question juridique ou plutôt comme une question économique posée par des juristes. Les représentants de l'industrie, eux, restaient fort discrets sur la question.

Peut-être cette discrétion était-elle due à un faible intérêt des grandes entreprises françaises pour le brevet d'invention. C'est ce que suggère en tous cas la comparaison du nombre de brevets pris par une société française comme Schneider et celui d'une société allemande comme Siemens. Entre 1865 et 1885 , la compagnie creusotine prit seulement onze brevets français en son nom tandis qu'entre 1870 et 1885 Siemens demandait 26 brevets en France. En 15 ans, la compagnie allemande avait pris deux fois plus de brevets en France que la compagnie française n'avait pu le faire chez elle en 20 ans. Un tel exemple expliquerait que les grands industriels aient quelque peu délaissé le débat sur la question des brevets aux professionnels de la propriété industrielle.

Ce monde des professionnels de la propriété industrielle présentait alors deux caractéristiques: il prétendait représenter avant tout les inventeurs indépendants et semblait fort divisé. Sans doute la tradition juridique française qui tendait à consacrer la personnalité de l'inventeur incitait-elle à défendre l'intérêt de l'inventeur individuel et à le présenter comme la victime d'un État désintéressé du progrès industriel et engoncé dans sa logique bureaucratique.

Ce point de vue, formulé maintes fois par différentes associations d'inventeurs, fut exprimé lors du congrès international des associations d'inventeurs en 1900, par M. Horsin-Déon, industriel et vice-président de l'Association des inventeurs et artistes industriels de Paris. Critiquant le désintérêt du gouvernement pour l'invention, Horsin-Déon condamnait la dette publique empêchant les inventions de réussir. Profitant du paiement des taxes sans aider les inventeurs, détournant les capitaux de l'invention, refusant de consacrer explicitement les droits de l'inventeur, l'État apparaissait à ses yeux comme un obstacle à la diffusion de l'innovation.

Alors qu'en Allemagne l'État laissait aux acteurs intéressés par la propriété industrielle le soin de trouver les compromis nécessaires à son évolution, en France les représentants des inventeurs parvenaient d'autant moins à entretenir un dialogue constructif avec l'administration qu'ils apparaissaient fortement marqués par les séquelles des vifs débats sur le libre-échangisme. L'opposition entre Louis Donzel, avocat à la cour d'appel de Paris, partisan du protectionnisme, et Eugène Pouillet, maître à penser des juristes en matière de propriété industrielle, fut à cet égard assez significative. Sans complexe, Donzel accusait l'Association française pour la protection de la propriété industrielle, dirigée par Pouillet, d'être "fondée par les grandes agences de brevets et les avocats que leurs intérêts professionnels solidarisent avec elles, $[\ldots]$ de se mettre en tête du mouvement de la réforme pour le contenir, le diriger et l'empêcher 
d'aboutir à la suppression des abus qui enrichissent les professionnels de la propriété industrielle $\aleph^{33}$.

Dès lors, les représentants de l'État étaient dans une position fort embarrassante. Confronté à un problème prenant une dimension nouvelle - la nécessité de réformer les cadres de la propriété industrielle - l'État ne put s'appuyer sur des interlocuteurs unis indiquant clairement une marche à suivre. Accusé par les uns de trahir la cause des inventeurs, poussé par les autres à maintenir un statu quo censé avoir fait ses preuves, l'État, embarrassé, était soumis à la tentation de l'immobilisme.

Ce ne furent que l'union de différents partenaires et la mise sur pied d'une coalition d'intérêts qui conduisirent l'État à prendre des mesures nouvelles comme l'illustrent la création de l'Office des brevets d'invention et des marques de fabrique en 1900 et l'adoption de la loi de 1902 . Toutes deux résultèrent d'une nouvelle attitude des milieux industriels apparue dans les années 1890. Pour faire face à la concurrence des puissances montantes, l'Allemagne et les États-Unis, il parut nécessaire de doter l'industrie d'infrastructures nouvelles permettant de s'assurer de la qualité des biens produits. C'est dans ce contexte, et suite à un partenariat entre l'État, la Chambre de commerce de Paris et la Société des ingénieurs civils, que fut réorganisé le Conservatoire national des arts et métiers, doté d'un laboratoire national d'essais rénové et centralisant en son sein les services de la propriété industrielle $e^{34}$.

La création de l'Office des brevets fut le résultat d'une entente entre le gouvernement, la Chambre de commerce et le Syndicat des ingénieurs-conseils. Par convention, la Chambre de commerce s'engageait à apporter un soutien financier à la réorganisation du Conservatoire, tandis que l'Etat lui garantissait une présence dans ses instances de décision, particulièrement au conseil technique de l'Office national des brevets. La convention fut entérinée par la loi du 9 juillet 1901 tandis qu'un décret du même jour créait le comité technique de l'Office composé notamment d'un représentant de l'Association française pour la protection de la propriété industrielle (AFPPI) et de deux membres de la Chambre de commerce. Les nominations au sein du comité assurèrent une large représentation du groupe de pression des juristes brocardés par Donzel: Claude Couhin, président de l'Association des inventeurs et artistes industriels, siégeait en qualité de jurisconsulte aux côtés de Pouillet, président de l'AFPPI ${ }^{35}$.

Incontestablement, la réforme fut largement facilitée par la détermination du ministre du Commerce et de l'Industrie, Alexandre Millerand, dont

\footnotetext{
${ }^{33}$ Chambre de commerce du Maine-et-Loire, extrait de la séance du 8 novembre 1899 , note demandée par M. le président à M. Louis Donzel. ACCIP, III.3.91 (07).

${ }^{34}$ Sur la réorganisation du CNAM, cf. Claudine FONTANON, Le Conservatoire national des arts et métiers (1794-1920), dans: Louis BERGERON et Patrice BoURDELAIS, La France n'est-elle pas douée pour l'industrie?, Paris 1998, p. 300-301 et notre thèse, Gabriel-David Galvez-Behar, Pour la fortune et pour la gloire. Inventeurs, propriété industrielle et organisation de l'invention en France (1870-1922), thèse de doctorat, Lille 2004, p. 295-341.

${ }^{35}$ Almanach national, Paris 1902.
} 
l'activité d'avocat et les liens d'amitié avec Couhin furent sans doute largement favorables au changement. La présence de ces groupes de pression au sein même d'un organisme d'État permit d'accélérer les tentatives de réformes. L'existence du comité technique explique sans aucun doute la facilité d'adoption de la loi du 7 avril 1902, modifiant la procédure de dépôt du brevet et atténuant les mécanismes de déchéance, demandes longtemps faites par les représentants des inventeurs.

Reste que les dispositions législatives et réglementaires créant l'Office des brevets (qui prendra plus tard le nom d'Office national de la propriété industrielle) ne suffirent pas à créer une institution répondant aux attentes des acteurs de la propriété industrielle. L'un des buts majeurs de ces derniers consistait en une centralisation en un seul lieu de toutes les démarches concernant le dépôt de brevets. Si l'installation de l'ONPI, en de nouveaux locaux, permit d'y abriter à la fois les instruments nécessaires aux recherches d'antériorités et au traitement des demandes, le paiement des taxes devait être fait à la recette principale, place Vendôme, tandis que les demandes de brevet pour Paris devaient être déposées à la préfecture de la Seine. Plus de cinq années furent nécessaires pour que le ministère des Finances acceptât de détacher un service de trésorerie à l'ONPI et seule l'intervention de Millerand, au détour de la discussion budgétaire de décembre 1908, permit d'y rapatrier les services de la préfecture.

La définition des prérogatives de l'ONPI montre combien la construction des missions de l'État est progressive et met en lumière ses mécanismes. En matière de propriété industrielle, force est de constater qu'en France comme en Allemagne l'action de l'État en matière de brevets d'invention n'est pas impulsée par l'Administration. Cette dernière est réclamée par des acteurs économiques qui ressentent la nécessité de se munir d'institutions nouvelles propres à assurer leur expansion économique. En Allemagne, ce sont les acteurs industriels qui intervinrent directement d'autant que l'État leur donna plus volontiers la parole et qu'il formalisa les cadres de leurs débats en la matière. L'évolution des cadres de la propriété industrielle en France semble répondre, quant à elle, à une autre logique. Ce sont les acteurs de la propriété industrielle - et non les industriels eux-mêmes - qui donnèrent les impulsions décisives. Se posa, dès lors, le problème aigu de leur représentativité et de leur légitimité à parler au nom des intérêts de l'industrie ou des inventeurs. Du fait de cet écart, leurs propositions furent toujours susceptibles d'être perçues comme des agissements intéressés qui ne pouvaient être cautionnées par l'Administration elle seule garante, bien entendu, de l'intérêt général. Il fallut attendre que soit réglée cette question pour que de nouveaux compromis puissent enfin être trouvés ${ }^{36}$.

${ }^{36}$ Sur les problèmes de représentativité des intérêts de la société civile, cf. Alain CHATRIOT, La démocratie sociale à la français. L'expérience du Conseil national économique, 1924 1940, Paris 2002. 
Administrer la propriété industrielle

L'intervention de l'État en matière de propriété industrielle ne repose pas uniquement sur de nouvelles conceptions juridiques; elle est actualisée par des pratiques concrètes qui trahissent aussi les contours et le fonctionnement de l'État.

\section{L'ONPI, administration modeste}

Afin de respecter, formellement au moins, les dispositions de l'Union de Paris $^{37}$, le gouvernement avait mis en place, dès 1883, un service de propriété industrielle au sein du ministère du Commerce et de l'Industrie et avait entrepris la publication du Bulletin de la propriété industrielle et commerciale. Malgré cela, la grande majorité des agents de brevets reprochait à l'Administration de ne pas assumer ses responsabilités en matière de publication et de consultation des brevets. L'exiguité des salles de consultation, le caractère partiel et peu pratique de la publication des brevets constituaient de sérieux obstacles pour les recherches d'antériorité et pour la prise de brevets. L'État, garant du respect de la loi de 1844 , qui le contraignait à communiquer gratuitement les brevets d'invention, ne remplissait pas sa mission.

La création de l'ONPI en 1901 apparut donc comme un compromis entre l'État et les acteurs de la propriété industrielle pour voir assurées certaines tâches jugées indispensables au progrès technique. En outre, la création du comité technique, on l'a vu, permit que fussent mieux pris en considération un certain nombre d'avis émanant du monde de la propriété industrielle. Ainsi, des travaux de la commission technique résulta l'adoption de la loi du 7 avril 1902 qui obligea l'État à assurer une publication in extenso et par fascicules séparés des brevets d'invention. Reste que ces progrès furent limités par des contraintes budgétaires pesantes qui empêchèrent l'ONPI de se doter des locaux et des personnels suffisants alors même que son activité augmentait de manière rapide ${ }^{38}$.

Afin de desserrer l'étau d'un budget trop juste, les membres de la commission technique se mobilisèrent. Alors que l'État, en 1905, encaissait près de 3848965 francs de taxes sur les brevets d'invention, l'ONPI ne bénéficiait que de recettes assez maigres qui ne lui permettaient pas de s'acquitter de sa mission dans les meilleures conditions. Aussi le combat pour l'autonomie fi-

\footnotetext{
${ }^{37}$ Signé en 1883 , la Convention internationale pour la protection de la propriété industrielle, dite "Union de Paris«, tend. à créer une base commune entre les pays signataires. Sur ce point, cf. Yves Plasseraud, François SAVIGNON, Paris 1883. Genèse du droit unioniste des brevets, Paris s.d.

${ }^{38}$ Rapport de la commission technique sur le fonctionnement de l'Office national de la propriété industrielle (avril 1908-janvier 1909) Paris s.d. p. 35.
} 
nancière fut-il l'un des enjeux majeurs de son évolution, mais il fallut attendre les lendemains de la guerre pour obtenir un tel statut sans que le nouvel office puisse se voir affecter les importantes taxes d'annuités.

Les attributions de l'ONPI, en théorie, étaient doubles. En application de la loi de 1844, il avait pour mission essentielle de remplir sa fonction administrative en matière de suivi, d'enregistrement et de délivrances des brevets (mais aussi des marques, dessins et modèles). Par ailleurs, le comité technique devait conseiller le ministre du Commerce et de l'Industrie sur toutes les questions intéressant la propriété industrielle tout en supervisant l'action de l'office.

Les pratiques des membres de l'office contribuèrent pourtant à dépasser ces strictes attributions. Ainsi, afin de permettre aux agents de brevets ou aux inventeurs de réaliser les recherches d'antériorité essentielles à leurs démarches, l'ONPI s'était constitué en un véritable centre de documentation (ouvert le dimanche!) comprenant l'ensemble des brevets français, l'essentiel des brevets étrangers ainsi qu'une bibliothèque de plus de 15000 ouvrages. Par ailleurs, Georges Breton, directeur de l'office, envisagea à plusieurs reprises d'organiser avec l'aide du Syndicat des ingénieurs-conseils en propriété industrielle des séances de consultations juridiques pour les inventeurs démunis.

Les membres du comité, loin de s'en tenir à une conception trop restrictive de leur action, furent conduits, eux aussi, à agir hors des cadres tracés par la loi, en pratiquant un examen préalable honteux. En effet, au début du $\mathrm{XX}^{\mathfrak{e}}$ siècle, le gouvernement tendit à s'opposer à la délivrance de brevets d'invention »contraire aux bonnes mœurs«. Par l'intermédiaire du comité technique, mais aussi du comité consultatif des arts et manufactures, l'État procédait à l'examen de certains brevets alors même que la loi réservait cette prérogative aux tribunaux, si besoin alertés par le ministère public. C'était là revenir sur l'équilibre trouvé en 1844 et confirmer, plus de cinquante ans plus tard, les craintes d'Odilon Barrot à l'encontre d'un possible conflit entre l'autorité administrative et les tribunaux ${ }^{39}$. Loin de constituer une fin en soi, l'institution se révéla donc être le lieu d'un débordement des cadres fixés par la loi et, de ce fait, un jalon posé pour forcer son évolution.

\section{Le Patentamt}

Face à l'administration française de la propriété industrielle, le Patentamt constitue une institution puissante et originale. Ses prérogatives et son influence résultent cependant de compromis et d'une évolution progressive $e^{40}$.

Siégeant à Berlin, le Patentamt constitua l'un des premiers offices fédéraux dont les membres étaient directement nommés par l'Empereur. L'un des aspects originaux de la création du Patentamt résida sans doute dans la coexistence de membres permanents et de membres non-permanents, "versés dans

${ }^{30}$ HUARD, Répertoire, p. 281.

${ }^{40}$ Sur le Patentamt de 1877, cf. KURZ, Das kaiserliche Patentamt. 
une branche de la science «, chargés de l'examen des brevets ${ }^{41}$. Bénéficiant d'un régime dérogatoire par rapport aux autres fonctionnaires de l'Empire, les membres non-permanents du Patentamt se virent reconnaître le droit de cumuler leur fonction au sein de l'office avec une autre profession et il leur fut permis de déposer des demandes de brevets.

Bien entendu, une telle situation était propice aux conflits d'intérêts. L'exemple le plus connu d'une telle situation fut celui de Werner Siemens qui siégea comme membre non-permanent à la section des nullités et des retraits de brevets. S'étant lui-même prononcé contre la possibilité de déposer des brevets pour un membre de l'office, ce dernier se trouva dans une situation délicate lorsque ses propres entreprises furent amenées à déposer des brevets. Après avoir proposé sa démission, qui fut refusée, Siemens sut vaincre ses scrupules et déposa une série de demandes ${ }^{42}$. La loi de 1891 n'apporta qu'un changement mineur à ces dispositions en substituant à la distinction "permanents «/»non-permanents« une distinction entre membres wjuristes« et membres »techniciens«, ces derniers se voyant autorisés à un cumul d'emploi. Cette dérogation devait permettre, comme précédemment, de faciliter le recrutement de membres auxquels le Patentamt ne pouvait assurer des appointements semblables à ceux offerts dans l'industrie privée ${ }^{43}$.

Le caractère dérogatoire de ces membres »techniciens" souligne bien l'absence de distinction tranchée entre le "public« et le "privé«" à l'intérieur d'une institution conçue pour être au service de l'industrie. L'office apparut alors comme une institution charnière entre l'État et l'industrie au sein de laquelle la frontière entre l'État et la "société civile« se trouvait brouillée. L'originalité du Patentamt se lit encore dans l'étendue de ses attributions que constituent la délivrance, la déclaration de nullité et le retrait des brevets. Déjà en 1877, la dénomination du Patentamt avait posé problème. Dans son projet, le gouvernement avait proposé de nommer "Patenthof « la nouvelle structure, mais le Conseil fédéral avait préféré le terme de »Patentamt« qui convenait mieux à une autorité administrative. Reste que cette ambiguitté entre autorité et juridiction administratives fut constitutive de l'identité de l'office. En effet, si l'examen préalable des demandes conduisait ce dernier à exercer les fonctions d'une autorité de police administrative, sa compétence à émettre des jugements sur les demandes d'annulation et de retrait lui donnait le caractère d'une véritable juridiction ${ }^{44}$.

À ces attributions administratives et même judiciaires s'ajoutait une attribution fiscale fort opportune. Contrairement au cas français, "l'Office des brevets présente, en effet, cette particularité très curieuse, qu'il a un budget autonome, dont les recettes, alimentées par les taxes qu'il perçoit, couvrent large-

${ }^{41}$ Conformément aux dispositions de l'article 13 de la loi de 1877 , cf. Loi sur les brevets d'invention du 25 mai 1877, dans: Annuaire de législation étrangère, 1878, p. 127.

${ }^{42}$ KURZ, Das kaiserliche Patentamt, p. 491.

${ }^{43}$ Sur ce point, cf. BONNET, Étude, p. 211.

${ }^{44}$ Charles Lyon-Caen définit le Patentamt comme sun tribunal et une autorité administrative«. Cf. LYON-CAEN, Loi sur les brevets, p. 126. 
ment les dépenses, avec un excédent annuel assez élevé qui rentre naturellement dans les caisses de l'État ${ }^{45}$. Cette particularité permit au Patentamt d'avoir les moyens d'assurer ses différentes missions. En 1910, ses recettes s'élevaient à plus de 10 millions de marks et ses dépenses à un peu moins de 5 millions dont l'essentiel était dépensé en frais de traitements. Grâce à ses ressources et du fait des attributions particulières qui lui étaient fixées, l'office allemand des brevets avait donné lieu au développement d'une bureaucratie assez imposante. Alors qu'en 1877 l'action du Patentamt reposait sur l'activité d'une trentaine de membres et d'employés, l'office de 1914 comptait 997 fonctionnaires ${ }^{46}$. En France, à la veille de la guerre, l'ONPI pouvait compter sur le dévouement d'une cinquantaine de fonctionnaires et d'employés.

Sans nul doute, le poids de ces ressources permit au Patentamt d'assumer pleinement une de ses missions essentielles au développement de l'industrie, la publicité de l'information technique et juridique concernant les brevets. Dès les années 1880 , le Patentamt consacra une bonne partie de son activité à la création de nouvelles publications: le Patentblatt diffusait l'information officielle concernant les brevets (les demandes, l'extinction des brevets, les annulations...) tandis que les Patentschriften, créées en 1880, publiaient partiellement le contenu des brevets d'invention. En 1894, le Patentamt publia un journal de jurisprudence ainsi que toute une série d'outils permettant de faciliter les recherches d'antériorité et de l'information technique. En 1914, il consacrait près de la moitié de son budget (hors traitement) à ses dépenses de publication.

Alors que l'ONPI apparaît comme un organisme essentiellement administratif, le Patentamt concentre des compétences variées à la fois administratives, judiciaires et fiscales qui lui permettent de répondre au mieux aux attentes supposées de l'industrie. Sans conteste, il fera figure d'exemple aux yeux d'industriels, de juristes et de fonctionnaires français soucieux de donner une nouvelle figure à l'État. Dans cette comparaison entre ces deux institutions, que les acteurs d'alors n'hésitaient pas à invoquer, se joue une nouvelle définition des missions de l'État. Dans un monde où la compétition entre les nations repose aussi sur l'innovation technique, l'État ne peut s'en tenir à son rôle de conservateur des équilibres sociaux et sa dimension protectrice, elle-même, ne suffit plus. De nouvelles structures sont alors nécessaires pour permettre à l'industrie d'assurer le développement de la nation grâce au développement des connaissances, grâce à la circulation et à l'appropriation de l'information technique. L'État les prend alors en charge, dans l'intérêt de tous.

Sans conteste, le mouvement d'industrialisation qui intervient à la fin du $\mathrm{XIX}^{\mathrm{e}}$ siècle repose sur l'émergence de nouveaux modes de production des connaissances et sur l'apparition de liens inédits entre la science et l'industrie. Réciproquement, grâce à l'essor des moyens de communication notamment, cette dynamique industrielle facilita grandement la circulation des savoirs et

\footnotetext{
45. BONNET, Étude, p. 240.

${ }^{\text {th }}$ KURZ, Das kaiserliche Patentamt, p. 490.
} 
accrut ainsi les possibilités d'emprunts mais aussi de contrefaçons et de dépossession. La compétition en matière d'innovation fut alors plus vive. Dès lors, les nations industrielles furent confrontées au développement d'une industrie favorisée par la diffusion des biens immatériels mais gênée par la difficulté de leur appropriation. Construire des normes nouvelles permettant d'encadrer cette concurrence apparut alors comme une nécessité.

Une telle tâche devait naturellement relever de l'État dans la mesure où ce dernier constitue le lieu de régulation où sont construits les compromis nécessaires à la vie collective, qu'elle soit politique, sociale ou économique ${ }^{47}$. Concilier les intérêts de l'inventeur et ceux de la collectivité, définir les contours de la propriété industrielle pour assurer le progrès voire la puissance de la cité se révèlent être des questions politiques, de véritables affaires de l'État.

Reste que le mode d'intervention de ce dernier n'est pas unique. C'est ce que permet de souligner notre comparaison en distinguant deux modèles d'intervention en matière de propriété industrielle. En France, prévaut longtemps un modèle fondé sur un héritage révolutionnaire sacralisant la personne de l'inventeur, sur une méfiance à l'égard de l'Administration et laissant à l'industrie le soin de distinguer d'elle-même les inventions qui lui seraient favorables. En Allemagne, c'est un modèle ad hoc qui s'affirme, construit pour répondre aux exigences du moment et conférant à l'État une responsabilité nouvelle. Deux conceptions de l'État semblent alors s'opposer: en France, un État conservateur, garant des droits de l'inventeur individuel; en Allemagne, un État >propulsif ‘, soucieux des intérêts de l'industrie.

Pour rendre compte de ces différences, bien des explications classiques pourraient être convoquées. Le manque de concentration de l'industrie française et une tradition slibéraler de l'État, issue de la Révolution, permettraient de comprendre les atermoiements du législateur en France. À l'inverse, la forte organisation du capitalisme allemand et le modèle d'un État allemand fort viendraient corroborer l'hypothèse d'un État interventionniste au service de l'industrie.

S'il n'est pas question de nier toute part de vérité à de telles affirmations, force est de constater qu'elles ne rendent guère compte des clairs-obscurs de la question. À maintes reprises, on l'a vu, l'existence de crises - au sens propre du terme, - d'interrogations, de remises en cause, montrent que les missions de l'État sont toujours susceptibles d'être redéfinies. Le choix de la nature de l'État s'inscrit toujours dans un champ ouvert, plein d'alternatives, même si les alternatives ne sont pas infinies et si le champ de la décision connaît certaines limites.

En effet, ce choix est contraint à la fois par des traditions et par des intérêts. Les traditions, qu'elles soient juridiques, culturelles, religieuses, interviennent pleinement et confèrent indéniablement une certaine inertie à l'évolution des figures de l'État. On a vu combien l'héritage de 1791 se fit sentir lors des dif-

${ }^{47}$ Cf. Jean BOUVIER, Le capitalisme et l'État en France, dans: Institut d'histoire économique et sociale de l'université de Paris I-Recherches et travaux 15 (1986) p. 47-63. 
férents débats portant sur l'évolution de la propriété industrielle en France. Certains intérêts, surtout lorsqu'ils sont liés à l'apparition de techniques nouvelles, parviennent cependant à rompre le cours de la tradition. L'exemple allemand, là, est parlant et souligne l'importance de la représentation des intérêts dans la construction des missions de l'État.

Aussi la question de l'intervention de l'État dans l'économie n'apparaît-elle pas comme une question uniquement économique. Trop souvent l'économie politique a tendance à réduire cette question à un simple problème de dosage: quel degré de protection pour la propriété intellectuelle, quel niveau d'impôt et de dépenses publiques sont donc nécessaires à la croissance? L'analyse historique vient casser l'automaticité des modèles ou des prévisions: elle met en lumière les hésitations et les renoncements, elle souligne les débats et les conflits qui se nouent autour de la définition de l'intérêt général et des intérêts particuliers. À sa lumière, le rôle économique de l'État réapparaît comme un problème essentiellement politique.

\section{Deutsche Zusammenfassung}

Die Geschichte des gewerblichen Urheberrechts, die hier am Beispiel des Patentes behandelt wird, betont den fast hoheitlichen Charakter des Urheberrechtsschutzes, der dem wirtschaftlichen Fortschritt dienen soll. Der Staat garantiert im Namen des Allgemeininteresses die Industrieentwicklung, indem er die Rechte des Erfinders schützt. Die Modalitäten der staatlichen Eingriffe in wirtschaftliche Angelegenheiten näher zu untersuchen, bedeutet, sich mehr oder weniger mit der Entwicklung eines fast unbekannten Aspekts der wirtschaftlichen Reglementierung auseinanderzusetzen.

Ein Vergleich zwischen Frankreich und Deutschland ist höchst aufschlußreich. Denn Frankreich hat den Ruf, auf diesem Gebiet ein Pionierland zu sein, da es seit 1791 eine moderne Gesetzgebung vorwies. Die französische Gesetzgebung, die 1844 durch die erste industrielle Revolution eine tiefgreifende Veränderung erfährt, erweist sich bezüglich des $\mathrm{Pa}$ tentschutzes über ein Jahrhundert hinaus als erstaunlich stabil. Die deutsche Gesetzgebung hingegen unterscheidet sich davon in zahlreichen Punkten: Mit der Verabschiedung eines neuen Gesetzes im Jahre 1877 erhält Deutschland eine wirkungsvolle Regelung, die auf einer vorangehenden, in Frankreich nicht üblichen Prüfung beruht.

Die Unterschiede zwischen den beiden gesetzlichen Regelungen spiegeln die verschiedenen Arten der Interessensvertretung von Industrie und Erfinder gegenüber dem Staat wider. Während in Deutschland die GroBindustrie und insbesondere die chemische Industrie ihr ganzes Gewicht einsetzt, um die Patentgesetzgebung zu beeinflussen, nehmen in Frankreich die Industriellen eher selten an solchen Debatten teil. Weitere Unterschiede kommen hinzu, etwa hinsichtlich der Institutionen selbst, die mit der Wahrung des gesetzlichen Urheberrechts beauftragt sind. Während Deutschland in Berlin das leistungsstarke Patentamt einrichtet, hat Frankreich trotz der Gründung des Office national de la propriété industrielle (ONPI) im Jahre 1901 Mühe, seine Urheberrechtsdienststellen umzustrukturieren.

Am Vorabend des Ersten Weltkriegs versucht man auf französischer Seite, sich von der deutschen Einrichtung inspirieren zu lassen, um die gesetzliche Regelung des gewerblichen Urheberrechts zu reformieren. Diese Versuche bleiben jedoch vergeblich, da das liberale Erbe des Gesetzes von 1844 noch mit seinem ganzen Gewicht nachwirkt. 